\title{
Labyrinthe
}

21 | 2005 (2)

Communauté en pièces : d'Europe, d'Islam et d'ailleurs

\section{Un certain universel de la communauté : l'invariant national}

\section{Marc Aymes}

\section{(2) OpenEdition \\ 1 Journals}

Édition électronique

URL : http://journals.openedition.org/labyrinthe/910

DOI : 10.4000/labyrinthe.910

ISSN : 1950-6031

Éditeur

Hermann

Édition imprimée

Date de publication : 22 juin 2005

Pagination : 61-64

Référence électronique

Marc Aymes, « Un certain universel de la communauté : l'invariant national », Labyrinthe [En ligne],

21 | 2005 (2), mis en ligne le 10 juillet 2008, consulté le 01 mai 2019. URL : http://

journals.openedition.org/labyrinthe/910 ; DOI : 10.4000/labyrinthe.910

Propriété intellectuelle 


\section{UN CERTAIN UNIVERSEL DE LA COMMUNAUTÉ: L'INVARIANT NATIONAL}

Marc AYMES

Tracer un trait d'union entre les identités confessionnelles d'hier et nationales d'aujourd'hui, n'est-ce pas aussi confondre les principes et les résultats de l'analyse? Si les catégories modernes de la nationalité semblent filles des «communautés » religieuses d'autrefois, ne seraitce pas simplement parce que l'on en est parti - parce que la communauté nationale a été posée en invariant, en universel de l'histoire des sociétés ? Par-delà la continuité recherchée entre des phénomènes historiques successifs, ce sont alors les catégories du savoir historique qui semblent relever d'un principe «communautaire» transcendant.

Revenons-en au trait d'union entre «communautés» confessionnelle et nationale $\rightarrow$ «La communauté d'historicité - La nation à l'horizon de la confession» $₫$ :il implique deux suppositions implicites. La première est que le mot «millet» renverrait, dans le monde ottoman (plus particulièrement ses dernières décennies), à une structuration politique et sociale effective, et déterminée par des critères autant «ethniques» que religieux. Je cite:

« La structure socio-ethnique et l'identité religieuse engendrées par le système de millet $^{1} »$;

«l'expérience historique des minorités ethniques et religieuses de l'empire [ottoman] $]^{2} » ;$

«la formation d'une autorité ethno-religieuse à la tête d'un ensemble ethnoreligieux ${ }^{3} »$.

1. Kemal Karpat, art. cit., p. 141.

2. Bruce Masters, op. cit., p. 1.

3. Athanasia Anagnostopoulou, art. cit., p. 154. 


\section{« Ethnicité »?}

Le concept, pour commode qu'il puisse être, n'est guère opératoire en l'absence d'un travail rigoureux de définition ${ }^{4}$. Je n'en retiens ici que la proposition suivante: «Nous utilisons les termes «ethnicité » et «groupe ethnique » pour désigner des phénomènes d'affiliation collective revendiquée ou attribuée, ainsi que des groupes qui se reconnaissent ou à qui on assigne une appartenance commune. Ces "groupes ethniques" peuvent exprimer des intérêts propres et se distinguer, dans un contexte où coexistent une pluralité de tels groupes, par des caractères culturels, linguistiques... distincts. Ces caractères peuvent être choisis comme emblèmes d'identité et de différence. II faut se garder de réifier ces catégories analytiques et éviter de les confondre avec des catégories "naturelles" de la réalité sociale ${ }^{5 .} » . \rightarrow$ « Communauté/groupe ethnique... »

Cependant, dans le domaine ottoman, le passage au crible ne semble pas s'être révélé concluant. À ma connaissance, les auteurs qui posent explicitement la question de la pertinence des dénominations ethniques dans l'Empire préfèrent s'en tenir à des remarques suspensives, sinon dubitatives: Metin Kunt argue certes de l'existence d'une certaine "solidarité ethnique et régionale » au sein des instances dirigeantes impériales au XVII siècle, mais ses conclusions privilégient l'horizon inter-individuel, et demeurent imprécises quant à l'hypothèse de "groupes " constitués suivant ces critères ethniques ${ }^{6}$. De son côté, Karl Barbir met en garde contre une interprétation ethnique de dénominations telles que Turc ou Arabe: il convient à cet égard d'effectuer "une importante distinction », souligne-t-il, afin «d'étudier des tendances culturelles et linguistiques plutôt que des associations ethniques et nationales potentiellement trompeuses ${ }^{7}$ ». Et puis, s'agissant des Balkans ottomans $d u X{ }^{\prime \prime I}{ }^{e}$ siècle, Paschalis $M$. Kitromilides donne une idée plus précise des enjeux de cette «distinction »-là : la diffusion de la "culture grecque» autorise sans doute, dit-il, à considérer celle-ci comme «un ingrédient d'une identité balkanique commune », mais

4. La question de l'ethnicité renvoie à des études aussi multiples qu'importantes, notamment dans le champ de l'anthropologie sociale : renvoyons simplement à Fredrik Barth (dir.), Ethnic Groups and Boundaries. The Social Organization of Culture Difference, Bergen-Oslo/Londres, Universitets Forgelet/George Allen \& Unwin, 1969; ainsi qu'à Philippe Poutignat, Jocelyne Streiff-Fenart (dir.), Théories de l'ethnicité, Paris, Puf, coll. « Le sociologue », 1995.

5. Pierre Centlivres et Micheline Centlivres-Demont, «Pratiques quotidiennes et usages politiques des termes ethniques dans l'Afghanistan du Nord-Est », dans Jean-Pierre Digard (dir.) Le Fait ethnique en Iran et en Afghanistan, Paris, CNRS, 1988, p.234.

6. «Ethnic-regional (cins) Solidarity in the Seventeenth Century Ottoman Establishment», International Journal of Middle East Studies 5 (1974), notamment p. 237 à propos des «cins groups ». 7. «From Pasha to Efendi : the Assimilation of Ottomans into Damascene Society, 1516-1783», International Journal of Turkish Studies 1/1 (1979), p. 68-69. 
non à lui imputer une «signification ethnique ${ }^{8}$ ». D'où une conclusion qui se rapporte directement à la question de la communauté, et de son rôle en tant que structure d'administration encadrée par des instances ecclésiastiques:

Les mesures d'administration ecclésiastique [...] devraient donc être interprétées en termes de politique ecclésiastique de l'époque, plutôt qu'au travers du prisme réfringent de conflits nationaux ultérieurs. [...] Le cœur de l'activité de l'Église orthodoxe était, par conséquent, la subsistance de la foi orthodoxe, non quelque projet présumé d'hellénisation ethnique de la population balkanique ${ }^{9}$.

De telles précautions, dira-t-on, s'appliquent à une époque antérieure à «l'ère du nationalisme ». Elles suffisent néanmoins à semer le trouble dans la longue durée des «minorités ethniques et religieuses » que Masters revendique; et, plus généralement, elles invitent à s'interroger sur la pertinence d'un lien trop étroit, trop automatique, entre les notions de communauté et d'ethnicité.

Or, quand bien même on admettrait l'effectivité des « communautés» confessionnelles dans l'Empire ottoman de l'époque (ce qui est une autre question $\rightarrow$ «Lin-coton : l'étoffe d'une communauté partagée » $\nleftarrow$ ), leur caractérisation en termes ethniques est loin d'aller de soi.

Venons-en à la seconde des suppositions annoncées, que l'on peut formuler ainsi : étant donné (première supposition) qu'il existe, au sein de la société ottomane, de tels critères «ethno-religieux» d'appartenance communautaire, c'est selon ces mêmes critères que se produirait, à «l'ère du nationalisme », la cristallisation d'identités nationales. Or, ce raisonnement ne revient-il pas à définir a priori la «communauté » d'avant l'âge des nationalismes à l'aide d'identifiants nationaux? En d'autres termes, le trait d'union semble tracé d'avance. Citons à nouveau Athanasia Anagnostopoulou, dont l'étude des « communautés » grecques d'Asie Mineure (millet-i R̂̂m), traduit bien cette tautologie de la nationalité : elle déclare étudier « le [sic] millet$i$ Rûm en tant qu'ensemble ethnico-religieux, à savoir national ${ }^{10}{ }$.

8. «Orthodox Culture and Collective Identity in the Ottoman Balkans during the Eighteenth Century », dans Kate Fleet (dir.), The Ottoman Empire in the Eighteenth Century, Oriente Moderno XVIII (n. s.), 1 (1999), p. 139.

9. Ibid., p. 142.

10. Athanasia Anagnostopoulou, op. cit., f. 813 
Aussi diverses qu'elles paraissent, ces communautés procèdent en dernier ressort d'un archétype national. Par où l'on voit que la communauté est investie de catégories d'interprétation déprises de toute historicité même, catégories dont l'invariance a été postulée au départ: à savoir implique, en l'occurrence, qu'un invariant national soit toujours déjà là.

Cette continuité-là, interprétative, assigne un certain style à l'écriture de l'histoire, un certain lieu à la «communauté» des savants: je serais tenté de dire, en paraphrasant la manière dont Anagnostopoulou caractérise l'Église orthodoxe chypriote, que l'universel national assume en dernier ressort un rôle de «seul et unique appareil d'organisation, de délimitation et de légitimation» du discours historien ${ }^{11}$. Ce n'est ici ni l' «organisation», ni la «délimitation», ni même la «légitimation» qui pose problème. Car l'histoire, si elle se mesure à l'étymologie des «archives» (arkhè) qu'elle sollicite, devine que son propos se situe quoi qu'il arrive «dans l'ordre du commencement aussi bien que dans l'ordre du commandement $»$ : bref, elle procède aussi d'un geste d'autorité, d'une «assignation à demeure ${ }^{12}$ ». Cela implique-t-il, pour autant, que la demeure soit «seul[e] et unique», et que l'assignation se conçoive comme un «appareil»: unicité exclusive, et automaticité mécanique ${ }^{13}$. Tel est l'enjeu d'une histoire des communautés: quelles continuités donner (plutôt que retrouver) à l'histoire, qui ne la transforment pas en une communauté exclusive ? $\Rightarrow$ «La communauté, un universel sociologique?»

11. Athanasia Anagnostopoulou, art. cit., p. 155.

12. Jacques Derrida, Mal d' archive. Une impression freudienne, Paris, Galilée, coll. « Incises », 1995, p. 12 et 13. Voir à ce propos Marc Aymes, «L'Archive dans ses œuvres (Rancière, Derrida)», Labyrinthe 17 (hiver 2004): Jacques Rancière, l'indiscipliné, p. 69-77.

13. Mon propos ne prétend plus, ce disant, commenter les auteurs cités (Anagnostopoulou en particulier), puisque son point de départ est un détournement de citation. Celui-ci cependant me semble valoir par l'interrogation qu'il permet de soulever. 